\title{
Recovery from adaptation as a function of stimulus orientation
}

\author{
CAROL S. FURCHNER and STEPHEN J. YOUNG \\ University of Colorado, Boulder, Colorado 80302
}

\begin{abstract}
These experiments examined the oblique effect in an adaptation paradigm. Reaction times (RT) to the presence of a grating test stimulus were obtained following adaptation to either a blank field or a grating of the same orientation as the test stimulus. Horizontal, vertical, and oblique test and adaptation orientations were employed. Test gratings were presented at several interstimulus intervals following offset of the adaptation stimulus. RTs following grating adaptation were elevated to a greater extent (relative to blank adaptation) for oblique then for horizontal or vertical stimuli, for two grating spatial frequencies. Differences in RT can be related to differences in sensitivity among channels responsible for detection of the various orientations.
\end{abstract}

In recent years, several studies have demonstrated that adaptation to a grating stimulus of a particular orientation in the picture plane markedly reduces sensitivity at that orientation. Sensitivity to other orientations is also reduced but to a lesser degree, the more deviant the test stimulus orientation is from that of the adapting stimulus (e.g., Blakemore \& Campbell, 1969; Gilinsky, 1968; Gilinsky \& Cohen, 1972; Houlihan \& Sekuler, 1968; Sekuler, 1965). These studies suggest that several different channels, each responding to a range of orientations, are used for processing stimulus orientation. The existence of these channels is consistent with physiological observations showing that primate visual cortex contains cells whose receptive fields are organized in such a way that a cell responds maximally to a single line orientation (e.g., Hubel \& Wiesel, 1968; Wurtz, 1969). Maffei, Fiorentini, and Bisti (1973) have shown neural adaptation of simple cortical cells which corresponds with some of the features of psychophysical adaptation.

Several lines of research suggest that the response of the human visual system differs with stimulus orientation. It has been established that the normal human observer is more sensitive to and capable of making tiner discriminations among stimuli presented near or at the vertical and horizontal visual axes than he is for oblique stimuli (see Appelle, 1972, and Taylor, 1963. for reviews). Appelle terms these differential orientation effects the "oblique effect." Campbell, Kulikowski, and Levinson (1966) have demonstrated that this effect is not simply a function of the optics of

Portions of this research were based on a master's thesis by C.S.F. at the University of Colorado. The research was supported by NIMH Predoctoral Fellowship 1 FO1 MH 54659-01. C.S.F. is now at the Physiological Laboratory, University of Cambridge, Cambridge. England. Requests for reprints should be sent to Stephen J. Young, Department of Psychology, University of Colorado, Boulder, Colorado 80302. the eye, that differential sensitivity and discriminability as a function of stimulus orientation are obtained when the refractive anomalies of the eye are corrected or bypassed. A few investigators (Andrews, 1967; Campbell \& Kulikowski, 1966) have suggested that the oblique effect is attributable to differences among the orientation channels in either sensitivity or bandwidth (the range of orientations to which a channel can respond), or both.

If orientation-specific channels differ in these or other characteristics, differences should be obtained in their response properties under adaptation. Gilinsky's (1968) data suggest that the duration thresholds for oblique test stimuli are greater following oblique adaptation than the corresponding duration thresholds for a horizontal test stimulus following horizontal adaptation or a vertical test stimulus following vertical adaptation, but direct comparisons were not made, and recovery functions were not obtained. Although some of the adaptation studies previously cited have employed different adaptation and test orientations, none have directly addressed the question of the oblique effect under an adaptation paradigm. In the present experiment, direct comparisons of the magnitude and the time-course of adaptation-produced changes in response were made among three orientations: $0 \mathrm{deg}$ (horizontal, $45 \mathrm{deg}$ (oblique), and $90 \mathrm{deg}$ (vertical, parallel with the body axis).

The effects of adaptation were evaluated using a reaction time (RT) measure. Preliminary research in this laboratory (Furchner, 1972) showed that the orientation specificity of the adaptation effect obtained using threshold measurement techniques could be obtained with response latency measurements: Reaction times are significantly elevated when adapting and test stimuli are presented at the same orientation, and not when the stimuli differ by 45 or $90 \mathrm{deg}$. Gilinsky and Cohen (1972) have found similar 
adaptation-produced elevations in RT. The results of their experiment are difficult to interpret, however. since high-contrast adapting and test stimuli of different luminance levels were used, separated by an ISI of $1 \mathrm{msec}$. Our observations indicate that these conditions are capable of generating brief but easily detectable retinal afterimages even when the adaptation stimulus is scanned (see below), which could differentially interfere with detection of a test stimulus depending on the difference in orientation of the adaptation and test stimuli. The techniques and stimulus conditions in the present experiments were selected in an attempt to overcome these methodological difficulties.

\section{EXPERIMENT I}

\section{Method}

Subjects. Four males, ranging in age from 18 to 33 , were used as subjects. Two subjects had Snellen acuity of $20 / 20$ or better without correction in the right eye, and two subjects wore glasses, giving them corrected acuity of $20 / 20$ in the right eye. The subjects were paid volunteers.

Procedure. On each trial, subjects monocularly (right eye) viewed an adaptation stimulus which was either a grating $(G)$ or an unpatterned field (B). The adaptation stimulus was presented for $10 \mathrm{sec}$, during which the subject scanned an octagonal pattern of dots, superimposed on the adaptation stimulus, at the rate of about one complete scan/second. Scanning was employed to minimize the formation of retinal afterimages of the adaptation grating which might have interfered with detection of the test stimulus. The superimposition of the scanning and grating fields under grating adaptation produced an adaptation stimulus with 0.56 Michelson contrast. A warning tone $(150 \mathrm{msec}, 1,000 \mathrm{~Hz})$ was sounded $450 \mathrm{msec}$ prior to the offset of the adaptation stimulus. The adaptation stimulus was followed by a blank ISI field, containing a centrally located fixation point. A test stimulus was superimposed on the ISI field at preselected intervals of 200 , $300,450,670,1,000,1,500,2,250,3,400$, and $5,100 \mathrm{msec}$ (approximately equally spaced on a logarithmic scale) following offset of the adaptation stimulus. A minimum ISI of $200 \mathrm{msec}$ was selected to avoid forward masking effects and to give the subject sufficient time to locate and accommodate the fixation point. Thus, test stimulus smearing due to accommodation or movement should have been minimized (Woll et al., 1970). The test stimulus was either a blank field or a low-contrast grating. Superimposition of the grating on the ISI field produced a grating test stimulus of 0.02 Michelson contrast. The subject was instructed to respond by pressing a telegraph key as soon as he detected the presence of a grating test stimulus ("see the stripes") and to withhold response if he saw no grating. Reaction time (RT) was measured from test stimulus onset. The test stimulus was turned off when the subject made a response or after $10 \mathrm{sec}$, whichever occurred first. A 10-sec intertrial interval (ITI) began with the offset of the test stimulus; the ITI field was identical with the ISI field. All conditions of the experiment were presented under a constant mean spatial luminance of $2.5 \mathrm{~mL}$.

Each session consisted of 66 trials, which were presented in six blocks of 10-12 trials each. The ISIs were randomized such that within each two blocks of trials, each ISI was presented twice, but a given ISI could occur 0,1 , or 2 times within each block of trials. This procedure prevented anticipation of a particular ISI within a block, and it ensured that all ISIs would be evenly distributed across the session. Within each block of trials, 1, 2, or 3 blank ("catch") trials were presented. Following each block of trials, the subject was given a 1-2-min rest period.
Six sessons were run per subject, on separate days. The grating adaptation and test stimuli were presented at orientations of $0 \mathrm{deg}$ (horizontal, H), +45 deg (oblique, O), and $+90 \mathrm{deg}$ (vertical, V, parallel to the body axis of the subject), in the following adaptation/test $(A / \Gamma)$ stimulus combinations, one per session: $\mathrm{H} / \mathrm{H}, \mathrm{B} / \mathrm{H}, \mathrm{V} / \mathrm{V}, \mathrm{B} / \mathrm{V}, \mathrm{O} / \mathrm{O}, \mathrm{B} / \mathrm{O}$. Reaction time to test stimuli under blank adaptation conditions controlled for the effects of foreperiod and provided a baseline measure of sensitivity to the test gratings. The order of presentation of these six sessions was randomized across subjects, with the constraint that for each test stimulus orientation, grating and blank adaptation conditions were presented on adjacent days. The order of blank and grating adaptation for a given test stimulus orientation was counterbalanced across subjects.

Prior to each session, each subject was given $5 \mathrm{~min}$ to adjust to the illumination levels at which the stimuli were presented. The experimental room was illuminated only by pilot lights from the equipment and a small light source near the experimenter.

Apparatus. Stimuli were presented in a four-channel tachistoscope assembly (Iconix: timer and clock-counter No. 6246; logic module No. 6711; lamp driver No. 6192; optical assembly No. 6137). Luminance and contrast were controlled by independently varying the current to fluorescent lamps illuminating each field. Lamp current was continuously monitored with a digital meter. Field 1 contained the scan pattern, Field 2, the grating adaptation stimulus; Field 3, the ISI and ITI field, contained the fixation point; Field 4 contained the test stimulus. All fields were partially occluded by concentric circular masks such that all stimuli subtended $4.8 \mathrm{deg}$ of visual angle. The tachistoscope was programmed to give the sequence of events described under Procedure. Reaction time was measured and the warning tone was generated by the tachistoscope circuitry.

Stimulus materials. Grating adaptation and test stimuli consisted of photographs of high-contrast square-wave gratings of a spatial frequency of 9 cycles/deg of visual angle, which could be rotated to $0,+45$, and +90 deg. Blank adaptation stimuli were constructed by replacing the grating stimulus with a blank card and resetting the luminance level to $2.5 \mathrm{~mL}$. Blank test stimuli were generated by switching off the grating test field. The ISI field luminance was electronically decremented whenever the grating test stimulus was on to maintain constant luminance. This small luminance decrement produced no discernible change in the lamp spectrum. An octagonal scanning pattern of eight small dots (octagon diameter approximately $2 \mathrm{deg}$ ) was superimposed on the adaptation field. These dots were positioned so that half fell on dark bars and half fell on light bars within the adaptation grating. Due to luminance summation, these dots appeared darker than the bars on which they were superimposed. A fixation point was superimposed on the ISI, test, and ITI fields.

\section{Results}

Geometric means of the RTs obtained on six trials within each ISI and A/T condition were computed for each subject. Completely repeated measures analyses of variance were performed separately on blank adaptation conditions and grating adaptation conditions. For grating adaptation conditions, $\mathrm{A} / \mathrm{T}$ orientation was significant $[\mathrm{F}(2,6)=6.18, \mathrm{p}<.05]$, as was ISI $[F(8,24)=19.91, p<.001]$. As shown in Figure 1, RT following briefer ISIs under grating adaptation was much greater than RT following long ISIs. The interaction between $\mathrm{A} / \mathrm{T}$ orientation and ISI did not approach significance $[F(16,48)=1.24]$.

Analysis of variance on RT to test stimuli presented under blank adaptation conditions yielded only a significant ISI effect $[F(8,24)=3.63, p<.01]$, which 


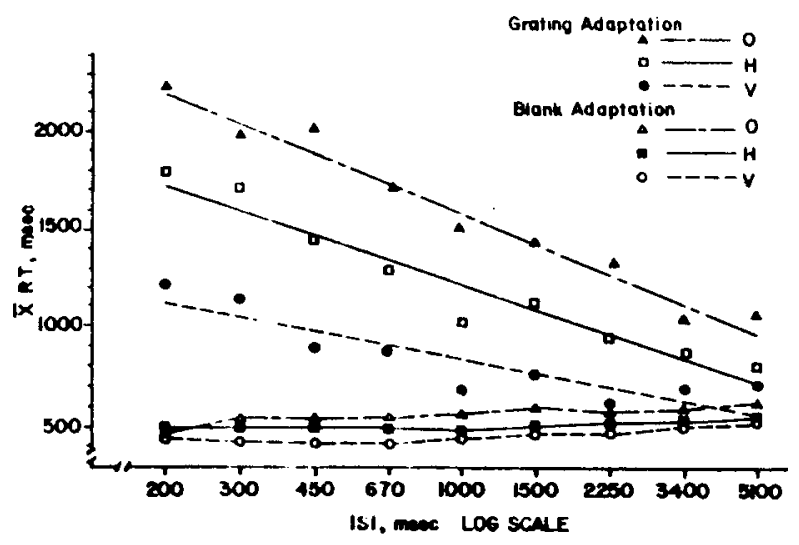

Figure 1. Mean RT as a function of adaptation condition, test orientation, and ISI in Experiment I. Lines were fit by least squares.

could be attributable to foreperiod effects on RT (Underwood, 1966); see Figure 1. Neither A/T orientation $[F(2,6)=1.83]$ nor the ISI by $A / T$ interaction $[F(16,48)=1.24]$ approached significance. Responses to the blank test field ("false alarms") were extremely infrequent in both grating and blank adaptation conditions; in each condition, subjects averaged less than one such response per session. Misses did not occur: subjects always detected the test stimulus within its $10-\mathrm{sec}$ presentation interval.

In order to obtain an estimate of the magnitude of the adaptation effect, the difference, $\Delta R T$, between RTs under grating and blank adaptation for each corresponding $\mathrm{A} / \mathrm{T}$ orientation, ISI, and subject was computed. Figure 2 shows $\Delta R T$ as a function of $A / T$ orientation and ISI (averaged group data). Analysis of variance on the $\Delta R T$ data again gave a significant effect of $\mathrm{A} / \mathrm{T}$ orientation $[\mathrm{F}(2,6)=5.92, \mathrm{p}<.04]$. Planned comparisons on the $A / T$ main effect indicated that $\Delta R T$ to oblique $A / T$ stimuli was significantly greater than $\Delta R T$ to vertical $A / T$ stimuli $[\mathrm{F}(1,3)=9.37, \mathrm{p}<.06]$ and $\Delta \mathrm{RT}$ to horizontal $\mathrm{A} / \mathrm{T}$ stimuli $[F(1,3)=8.82, p<.06]$, but $\Delta R T$ did not differ significantly between horizontal and vertical $[F(1.3)=2.08, p<.24]$. The effect of ISI was also significant $[F(8,24)=24.01, \mathrm{p}<.001]$, showing a general decrease in $\Delta \mathrm{RT}$ with increasing ISI. The overall interaction between ISI and $A / T$ orientation, however, was not significant $[\mathrm{F}(16,24)=1.07]$.

A regression analysis was performed using orthogonal polynomials on the $\Delta \mathrm{RT}$ data. The ISI values were treated as equally spaced on a logarithmic scale. The only significant component was the linear trend $[F(1,3)=43.38, p<.01]$, indicating that $\Delta R T$ changes linearly on a logarithmic time scale. The linear interaction, quadratic, quadratic interaction, and the pooled residual components did not approach significance. Best fit regresion lines were calculated

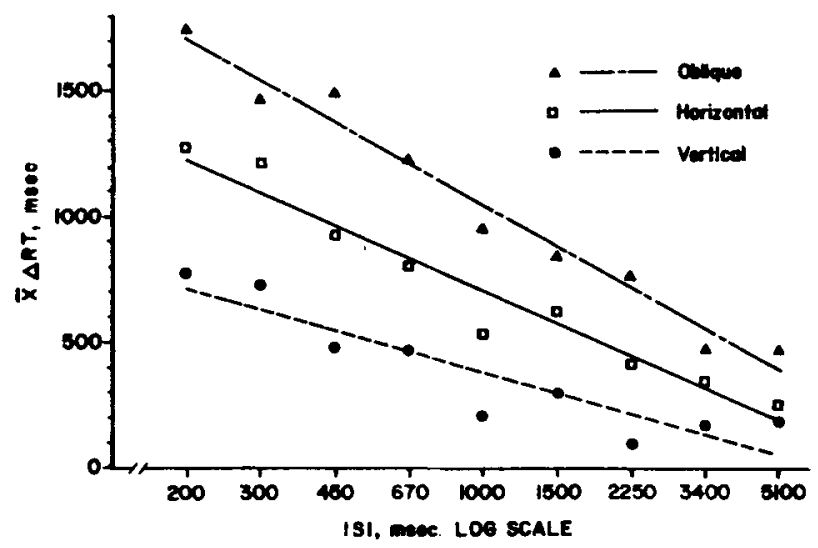

Figure 2. Mean $\Delta R T$ as a function of grating orientation and ISI in Experiment I. Lines were fit by least squares.

using the method of least squares for group means (Table 1) and individual subject means for each orientation condition according to the equation

$$
\Delta \mathrm{RT}=\mathrm{b}-\mathrm{a}(\log \mathrm{ISI})
$$

The lines shown in Figures 1 and 2 were derived from this equation. As shown in Table 1, this equation accounts for $83 \%-98 \%$ of the variance in group means [and $30 \%-98 \%$ (mean $=70 \%$ ) of the variance in individual subject means]. Extrapolated total recovery times (where $\Delta R T=0$ ) are also given in Table 1. A linear relationship between mean RT and $\log$ ISI was also found (accounting for $75 \%-96 \%$ of the variance of the group means) as shown in Figure 1. This suggests that the $\Delta R T$ equations are not artifacts of the subtraction.

\section{Discussion}

The results of this experiment indicate that the degree of adaptation, as measured by $\Delta \mathrm{RT}$, differs for the three $A / T$ orientations and that as time following adaptation is increased in terms of $\log$ ISI, $\Delta$ RT decreases at the same rate for these orientations. Thus, differences in total recovery times were obtained. Although differences in the magnitude of adaptation and recovery time may be

Table 1

Regression Equations Describing Recovery from Adaptation Over Time $(\Delta R T)$ for Group Means: Least Squares Fit

\begin{tabular}{|c|c|c|c|}
\hline Orientation & Equation & $\begin{array}{l}\text { Per- } \\
\text { cent } \\
\text { Vari- } \\
\text { ance }\end{array}$ & $\begin{array}{c}\text { Estimated } \\
\text { Total } \\
\text { Recovery } \\
\text { Time } \\
\text { (Sec) }\end{array}$ \\
\hline $\begin{array}{l}\text { Horizontal } \\
\text { Vertical } \\
\text { Oblique }\end{array}$ & $\begin{array}{l}\Delta R T=2952-747 \log \text { ISI } \\
\Delta R T=1772-464 \log \text { ISI } \\
\Delta R T=3866-937 \log \text { ISI }\end{array}$ & $\begin{array}{l}95 \\
83 \\
98\end{array}$ & $\begin{array}{r}8.95 \\
6.59 \\
13.36\end{array}$ \\
\hline
\end{tabular}


attributable to differences in the characteristics of the channels responsible for the detection of these three orientations, alternative explanations must be considered.

Although Campbell et al. (1966) have demonstrated that the oblique effect is not merely attributable to astigmatism, this anomaly could have contributed to the effect obtained in the present experiment. If an astigmatic subject is accommodated for detection of one axis, the astigmatism would reduce the contrat of a grating at the defocused axis (Blakemore \& Nachmias, 1971) or make it more difficult for the subject to detect the test grating at this axis (Blakemore, Muncey, \& Ridley, 1971). Three subjects were found to be slightly astigmatic under the viewing conditions of this experiment so that either a near-vertical or near-horizontal axis was seen less clearly than any other axis. Adaptation was more effective and recovery less rapid for stimuli presented along the poorest axis, but in no case were $\Delta R T$ s to the poorer axis as large as those found for oblique stimuli. The fourth subject had no astigmatism we could detect; his $\Delta R T$ s to horizontal and vertical were nearly identical, but clearly less than those for oblique. Although the presence of slight astigmatism in some subjects clearly increased the overall variability of the data, ${ }^{1}$ these results suggest that while astigmatism may be a factor in adaptation, it does not seem to account for the greater effect of adaptation to oblique stimuli. Replication of the experiment on emmetropic subjects, however, is desirable (see below).

Even though subjects were given instructions to scan continuously throughout the adaptation interval in order to forestall afterimage formation, it is possible that the duration of the last fixation of scan might have been sufficient for weak afterimage development. If afterimages were formed with equal magnitude in each condition, they would tend to affect RT at short ISIs more than at long ISIs, but, while not desirable, their presence would not seriously affect the interpretation of the results. If, however, for some reason, afterimages were formed differentially with respect to adapting orientation, their presence would cast serious doubts on a systems-difference interpretation of the experimental results.

Finally, it was suggested that the results of these experiments could be due to some form of nondifferential forward masking, and that a noise adaptation stimulus should be employed as a control. This effect seemed unlikely, at the ISIs and adapting stimulus durations employed. Generally, it appears that the masking largely depends on the degree to which the test stimulus and mask share frequency and orientation components (and the relative amplitudes of components of each spectrum) (e.g., Weisstein \& Bisaha, 1972). For example, Stromeyer and Julesz (1972) and Carter and Henning (1971) have shown that visual noise is effective in increasing thresholds for detection of a grating only when the grating and mask have spatial frequency (and orientation) components in common. Experiments (e.g., Houlihan \& Sekuler, 1968) in which mask orientation is varied demonstrated that contour masking effects are equivalent to blank masking effects when the contour orientations differ by $42 \mathrm{deg}$.

The issue may be argued from another point of view. If foreward masking is thought to occur through some interference in processing, the presentation of the mask should interfere with processing of the test stimulus up to some mask duration (mask processing time). As mask duration is further increased, processing interference should no longer occur and only a small component of interference should remain from luminance summation (Kahneman, 1967). If further interference is obtained with additional increases in mask duration, this would suggest that the analyzer itself is rendered inoperative, i.e., adapted.

Evidence for processing interference operating over a short time range was found by Houlihan and Sekuler (1968). Sensitivity following presentation of grating masks of 50 and $500 \mathrm{msec}$ duration did not differ significantly (for a same-orientation test stimulus), whereas sensitivity following a 5-msec mask exposure was considerably greater than that obtained following 50- and 500-msec mask durations. Since the ISI was $10 \mathrm{msec}$, this suggests that processing interference effects did not extend beyond an SOA of 60 msec.

Even though masking effects appear to operate over shorter ISIs than those employed, it is possible that masking effects could have contributed to the RT elevations obtained in these experiments. It is an empirical question whether or not such effects might have extended beyond the minimum ISI of $200 \mathrm{msec}$. A more critical issue is whether or not such potential masking effects might have been the source of the "oblique effect" obtained. If, for some reason, oblique detection systems were more sensitive to contour masking effects than vertical or horizontal systems, then could the observed greater increase in RT be a function of such masking and not due to processes of adaptation, per se? Also, if masking effects were present but nondifferential, they might appear differential due to some sensitivity difference between oblique vs. horizontal and vertical detecting systems.

In order to evaluate the possible contributions of astigmatism and afterimages, Experiment II was performed. It consisted of a partial replication of Experiment I on an emmetropic subject. Adaptation stimuli were selected according to procedures discussed below to meet the criterion that they generated no detectable afterimages over the range of 
ISIs employed. Experiment III describes the results of a test of the hypothesis that nonspecific masking contributed to the RT elevations obtained in these experiments and differentially affected RT as a function of test stimulus orientation.

\section{EXPERIMENT II}

\section{Method}

Subject. A single emmetropic ${ }^{2}$ subject (male, age 26 ) was used.

Stimulus materials. Higher frequency gratings (12 cycles/ $/ \mathrm{eg})$ were used for the adaptation and test stimuli than those in Experiment I to minimize the persistence of afterimages (Brindley, 1962). The contrast of the adapting stimulus was adjusted to a value of 0.20 , at which no afterimages could be detected, over the range of ISIs employed. The presence of afterimages was assessed by the following procedures. To maximize generation of afterimages, the subject was instructed to fixate on a dot in the center of the field, and the adaptation grating was flashed (mean spatial luminance of $2.5 \mathrm{~mL}$ ) for $500 \mathrm{msec}$. Following an ISI of $100 \mathrm{msec}$ (shorter than the minimum experimental ISI), a second 12-cycle/deg grating, rotated $15 \mathrm{deg}$ with respect to the adapting grating, was flashed for $1 \mathrm{sec}$. The subject was requested to report if he saw a moiré pattern, the presence of which would indicate that an afterimage of the adaptation grating was present and superimposed on the second grating. If no moire pattern was seen, then it was probable that the subject had no afterimage. The adapting stimulus contrast was decreased until the subject reported no moiré patterns out of 25 trials, and was set at 0.20 . As a further check, the subject was asked to report on the presence of moiré patterns under the conditions of the experiment, following scanning of the adaptation grating for $5 \mathrm{sec}$ at an ISI of $200 \mathrm{msec}$. An elliptical scan pattern, with major axis perpendicular to the grating and subtending $1.95 \mathrm{deg}$ of visual angle, was used, since the subject reported having least difficulty in scanning this type of pattern at any orientation. For all orientations used in Experiment II, no moiré patterns were reported in this final check.

Apparatus. The same apparatus was used as in Experiment I.

Procedure. The sequence of events within a trial and the task of the subject were identical with those of Experiment I. Grating adaptation and test stimuli were again presented only at the same orientations; four $A / T$ orientations, $0,+45,90$, and $-45 \mathrm{deg}$, were presented respectively, on separate days. Within each sessions, 20 blocks of 6-8 trials were given; in odd-numbered blocks, grating adaptation stimuli were presented, and in even-numbered blocks, blank adaptation stimuli were presented. ISIs of $200,300,450,670$, and $1,000 \mathrm{msec}$ were presented in separate blocks, such that adjacent grating and blank adaptation blocks were presented at the same ISI, and block order was randomized and counterbalanced with respect to ISI within each session. Each block contained 5 trials in which a grating test stimulus was presented, and 1-3 trials in which a blank ("catch") test stimulus was presented, giving 10 R'Ts per ISI for each grating and blank adaptation stimulus.

Table 2

Best Fit Equations for $\Delta \mathbf{R T}^{*}$

\begin{tabular}{ccc}
$\begin{array}{c}\text { Orientation } \\
\text { (Deg) }\end{array}$ & Equation & $\begin{array}{c}\text { Percent } \\
\text { Vari- } \\
\text { ance }\end{array}$ \\
\hline 0 (Horizontal) & $\Delta R T=574.0-177.2 \log$ ISI & 98 \\
90 (Vertical) & $\Delta R T=486.5-139.9 \log$ ISI & 99 \\
+45 (Right) & $\Delta R T=588.8-168.7 \log$ ISI & 93 \\
-45 (Left) & $\Delta R T=633.2-178.1 \log$ ISI & 92 \\
\hline
\end{tabular}

${ }^{*}$ Experiment II, one subject, ISI in milliseconds.

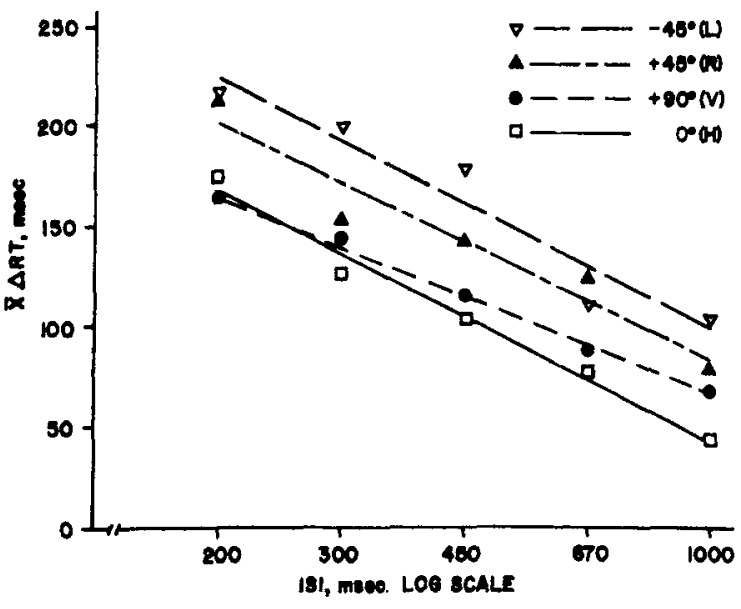

Figure 3. Mean $\triangle R T$ as a function of grating orientation and ISI for the subject of Experiment II. Lines were fit by least squares.

\section{Results}

As in Experiment I, $\Delta R T$ scores were computed for each ISI and $A / T$ condition, and are shown in Figure 3. Analysis of variance on this data, using first vs. second half of sessions as the source of error terms, revealed a significant $A / T$ orientation effect $[F(3,3)$ $=10.50 \mathrm{p}<.05$ ]. The $\Delta \mathrm{RT}$ s were greater for oblique stimuli than for horizontal and vertical stimuli, which is consistent with Experiment I. The $\Delta \mathrm{RT}$ s for left oblique stimuli were also greater than those for right oblique, which is consistent with the slight contrast threshold differences found for this subject for these axes. A significant ISI effect $[F(4,4)=116.44$, $\mathrm{p}<.001]$ was also found. The $\mathrm{A} / \mathrm{T}$ by ISI interaction was not significant $[F(12,12)<1]$. These results agree strongly with the results of Experiment I. Equations for the regression lines shown in Figure 3 are given in Table 2 .

\section{EXPERIMENT III}

\section{Method}

Subject. The experiment was conducted on a single emmetropic subject (male, age 34 ).

Procedure. The sequence of events within a trial and the task of the subject were identical with those of Experiments I and II. Test stimuli were presented at 0,45 , and $90 \mathrm{deg}$, after adaptation to gratings which formed angles of 0,45 , or 90 deg with each test stimulus, two noise stimuli, or a uniform field. These six adaptation stimulus conditions were orthogonally combined with the three test stimulus orientations and two ISIs ( 200 and $450 \mathrm{msec}$ ). A single test stimulus orientation was presented in each of six sessions. Each test stimulus was presented in two sessions, in counterbalanced order. The adapting stimulus conditions were presented in blocks of 14-16 trials each. Within each block, the ISIs were randomly ordered and 2-4 blank test trials were presented. The order of adapting stimulus presentation was randomized and counterbalanced across sessions. This procedure yielded $12 \mathrm{RTs}$ per condition. In addition, $\mathrm{RTs}$ were obtained following presentation of the noise-adapting stimuli with a 5-msec ISI. 


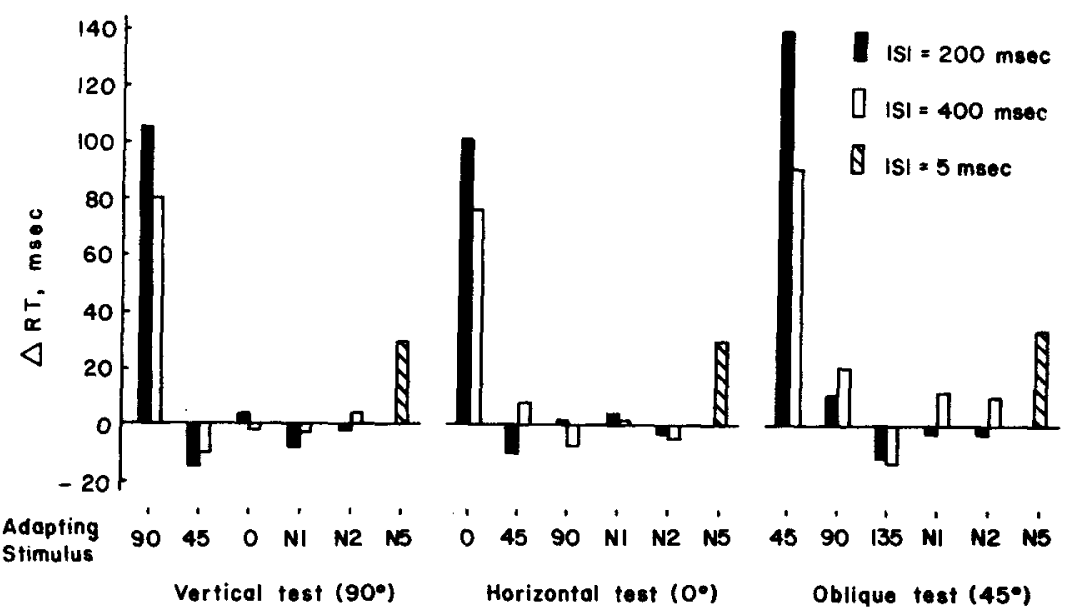

Figure 4. Results of Experiment III, showing the RT elevation ( $\triangle R T)$ obtained under six patterned adaptation conditions. Mean reaction times following uniform field adaptation were: Vertical test, ISI $=5 \mathrm{msec}$ 479 msec; ISI $=200: 400$ msec; ISI $=450$ : 366 msec. Horizontal test, ISI $=5$ msec: 500 msec; ISI $=200: 393 \mathrm{msec}$; ISI $=450$ : 361 msec. Oblique test, ISI $=5$ msec: 548 msec; ISI $=200: 480$ msec; ISI $=450$ : 466 msec. Grating adapting stimull formed angles of 0,45 , and $90 \mathrm{deg}$ with the test stimuli. N1 was patterned visual noise, described in the text. N2 was the same stimulus rotated 45 deg from N1. N5 represents a noise adapting stimulus, $\mathrm{N1}$, presented with a 5-msec ISI.
Apparatus. The same apparatus was used as in Experiments I and II.

Stimulus materials. Grating stimuli, contrast levels, and luminance levels were identical with those used in Experiment II. The noise stimulus consisted of randomly spaced dots and lines. The dots, lines, and spaces varied in size from approximately twice to one-fourth the width of one grating cycle. The lines also varied in length and orientation. Thus, the mask contained a broad spectrum of orientation and spatial frequency components. The contrast of the noise mask was set equal to the contrast of the gratings, at 0.20 . Prior to the adaptation experiment, the effectiveness of the mask was determined by measuring duration thresholds for the test stimuli in which the sequence of events was as follows: 200-msec mask, 5-msec ISI, test stimulus of variable duration. A staircase procedure was used, with $30 \%$ blank test stimuli. The masking stimulus produced increases in duration threshold over those obtained with a homogeneous mask of about $100 \mathrm{msec}$ (for $70 \%$ correct detection). About $1 \%$ false alarms were obtained.

\section{Results}

A 6 by 3 by 2 by 2 analysis of variance was performed on the geometric means of the six RTs obtained for each adaptation, test, ISI, and session condition combination. Session interactions were used as error terms. The analysis of variance yielded a signiticant main effect of adaptation condition (A) $[\mathrm{F}(5.5)=112.21, \mathrm{p}<.001] ; \mathrm{RT}$ was elevated only when the adaptation stimulus was a grating and of identical orientation of the test stimulus. The effect of ISI was also significant $[\mathrm{F}(1,1)=791.51, \mathrm{p}<.025]$, as was test stimulus orientation $(\mathrm{T}([\mathrm{F}(2,2)=286.81$, $\mathrm{p}<.005]$. RTs were shorter at $450 \mathrm{msec}$, and were greater for oblique than for horizontal or vertical adaptation. The A by $\mathrm{T}$ interaction $[\mathrm{F}(10,10)=3.14$, $\mathrm{p}<.05]$ and the A by ISI interaction $[F(5,5)=5.11$, $\mathrm{p}<.05$ ] were also significant. These two-way interactions may be interpreted as follows: For the $\mathrm{A}$ by T, RTs were elevated for oblique same-orientation adaptation conditions more than under the corresponding conditions for horizontal and vertical stimuli. For the A by ISI interaction, the variable of ISI had little systematic effect on RT under all adapting conditions except same orientation. The ISI by $T$ interaction approached significance $[\mathrm{F}(2,2)=$ $9.29, \mathrm{p}<.10$ ], while the $\mathrm{A}$ by ISI by $\mathrm{T}$ interaction was not significant $[F(10,10)<1]$.

$\triangle R T$ scores, shown in Figure 4, were computed by subtracting RT scores obtained under patterned adaptation from RT scores obtained under uniform field adaptation. As can be seen, neither the noise adapting stimuli nor the different orientation grating stimuli had an appreciable effect on RT. The only exception, not included in the above analysis, occurred when a noise stimulus preceded the test stimulus by an ISI of $5 \mathrm{msec}$. Here, RT elevations of 30-35 msec were obtained.

\section{GENERAL DISCUSSION}

The results of Experiment II replicate those of Experiment I, to the extent that neither astigmatism nor differentially persistent afterimages appear to account for the result that adaptation produces effects of greater magnitude when adaptation and test stimuli are oblique than when they are horizontal or vertical. Logarithmic expressions of recovery from adaptation describe the change in $\Delta \mathrm{RT}$ over time in Experiment II with a fairly high degree of precision. Although retinal afterimages may have contributed to $\Delta R T$ scores in Experiment $I$, this latter result suggests that their contribution was small in comparison with the decline in sensitivity produced by adaptation. The fact that $\Delta R T$ s were generally lower in Experiment II than in Experiment I may be attributed to the lower contrast of the adaptation stimulus (Blakemore \& Nachmias, 1971), the shorter adaptation duration (Gilinsky \& Cohen, 1972) employed in Experiment II, and the somewhat puzzling but fairly prevalent existence of large individual differences in sensitivity decline following adaptation at any orientation.

From Experiment III, it is clear that an adaptation stimulus differing by 45 or $90 \mathrm{deg}$ from the test 
stimulus orientation, or consisting of noise. does not have an appreciable effect on RT to the test stimulus. Furthermore, a noise stimulus does not have a differential effect with respect to test stimulus orientation. Although it is possible that masking effects might contribute to apparent sensitivity losses when very brief ISIs are used, it seems clear that such effects were not obtained under the present experimental conditions. It is difficult to determine whether the 30-msec RT elevation obtained at the 5-msec ISI following noise adaptation was produced by masking or slight adaptation.

The results of these experiments suggest that the mode of processing spatial information within each of the orientation channels is similar, but that the channels differ in certain parameters. The present results suggest that the magnitude of the sensitivity decline obtained under same-orientation adaptation is related to the sensitivity differences among the channels previously observed in other investigations under blank adaptation. RT elevations obtained for oblique same-orientation adaptation were consistently greater than the corresponding elevations for horizontal and vertical adaptation. That is, the time required to achieve sufticient channel output for detection, following adaptation, is greater for oblique channels. A channel may be conceptualized as having an analyzing component (sensitive to stimulus orientation) and a component which integrates the output of the analyzer over some small time inveral. If it is presumed that detection is dependent on achieving sufficient integrated analyzer output, then the RT differences obtained under adaptation could reflect differences in analyzer sensitivity or in channel integration characteristics.

Three general models have been proposed to account for sensitivity differences in terms of channel output. One (Bouma \& Andriessen, 1970) postulates that channels for the various orientations differ in peak sensitivity (e.g., channel output to an optimum stimulus) but have the same bandwidth (range of orientations to which they respond). Another (Andrews, 1967) suggests that channels have equal peak sensitivities, but different bandwidths, and this latter factor produces apparent differences in orientation sensitivity when measured psychophysically. A third (Appelle, 1972) proposes that the channels are unequally distributed, with more channels sensitive to major axes than oblique axes. Another possibility. suggested by the present study, involves differences in integration characteristics among the channels; these differences might be translated neurophysiologically into greater convergence of input or greater inhibition-produced signal-to-noise maximization for vertical and horizontal channels. None of these explanations would be inconsistent with the possibility of experiential origins of differential sensitivity, as has been suggested by some investigators (e.g.. Blakemore d Cooper, 1970; Mitchell, Freeman, Millodot. \& Hategstrom. 1973). Since the sources of differential sensitivity are not known, it would seem that further research in this area, whether or not directed at specifically testing one or another of these models, would be more fruitful in terms of its own generality and in terms of elucidating the sources of differential sensitivity, if direct comparisons among response at a variety oi orientations were made.

\section{REFERENCES}

ANDREws, D. P. Perception of contour orientation in the central fovea. Part I: Short lines. Vision Research, 1967, 7, 975-997.

Appelle, S. Perception and discrimination as a function of stimulus orientation: The "oblique effect" in man and animals. Psychological Bulletin, 1972, 78, 266-278.

BlaKemore. C., \& CAMPBEll, F. W. On the existence of neurones in the human visual system selectively sensitive to the orientation and size of retinal images. Journal of Physiology (London), 1969, 203, 237-260.

Blakemore, C., \& Cooper, G. E. Development of the brain depends on the visual environment. Nature (London), 1970. 228. 477-478.

Blakemore, C., Muncey, J. P., \& Ridley, R. M. Perceptual fading of a stabilized cortical image. Nature (London), 1971. 233. 204-205.

Blakemore, C., \& NaChmias, J. The orientation specificity of two visual aftereffects. Joumal of Physiology (London), 1971. 213, 157-174.

Bouma, H., \& ANDriessen, J. J. Induced changes in the perceived orientation of line segments. Vision Research, 1970, 10. 333-349.

Brindley, G. S. Two new properties of foveal afterimages and a photochemical hypothesis to explain them. Journal of Physiology (London). 1962. 164. 168-179.

Campbell, F. W., \& Kulikowski, J. J. Orientational selectivity of the human visual system. Journal of Physiology (London). 1966, 187, 437-445.

Campbell, F. W., Kulikowskr, J. J., \& Levinson, J. The effect of orientation on the visual resolution of gratings. Journal of Physiology (London), 1966, 187, 427-436.

Carter, B. E., \& Henning, G. B. The detection of gratings in narrow-band visual noise. Journal of Physiology (London), 1971. 219. 355.365.

FURChNER, C. S. Temporal parameters of recovery from orientation-specific adaptation. Unpublished MA thesis, Department of Psychology, University of Colorado. Boulder, 1972.

Gilinsky, A. S. Orientation-specific effects of patterns of adapting light on visual acuity. Joumal of the Optical Society of America. 1968, 58, 13-18.

Gilinsky, A. S., \& Cohen, H. H. Reaction time to change in visual orientation. Perception \& Psychophysics, 1972, 11. 129-134.

Houlihan, K., \& Sekuler, R. W. Contour interactions in visual masking. Journal of Experimental Psychology, 1968. 77. $281-285$.

Hubel, D. H., \& Wresel, T. N. Receptive fields and functional architecture of monkey striate cortex. Joumal of Physiology (London). 1968, 195, 215-243.

Kahneman, D. An onset-onset law for one case of apparent motion and metacontrast. Perception \& Psychophysics, 1967. 2. $557-583$.

Maffei, L., Fiorenrini, A., \& Bisti, S. Neural correlate of perceptual adaptation of gratings. Science, 1973, 182. 1036-1038. 
Mitchell, D, E., Freeman, R. D., Millodot, M., \& Haegstrom, G. Meridional amblyopia: Evidence for modification of the visual system by early experience. Vision Research. 1973, 13. 535-558.

SeKuler, R. W. Spatial and temporal determinants of visual backward masking. Journal of Experimental Psychology, 1965 , 70. $401-406$.

Southall. J. P. C. Introduction to physiological optics. New York: Dover. 1937.

Stromeyer, C. F.. III, \& Julesz, B. Spatial frequency masking in vision: Critical bands and spread of masking. Journal of the Optical Society of America, 1972, 62, 1221-1232.

TAYLOR, M. M. Visual discrimination and orientation. Journal of the Optical Society of America, 1963, 53, 763-765.

UNDERWOOD, B. J. Experimental psychology. New York: Appleton-Century-Crofts, 1966.

Weisstein. N.. \& Bisaha, J. Gratings mask bars and bars mask gratings: Visual frequency response to aperiodic stimuli. Science, 1972, 176. 1047-1049

Woll, S., ERIKsen, C. W., \& HaKe, H. W. A forced-choice study of edge detectors in the human visual system.Perception \& Psychophysics, 1970, 9, 247-252.

WURTz, R. H. Visual receptive fields of striate cortex neurons in awake monkeys. Journal of Neurophysiology, 1969, 32. 727-742.

\section{NOTES}

1. In agreement with others (e.g., Woll, Eriksen. \& Hake, 1970), we find large individual differences in response following adaptation, both in terms of horizontal-vertical response differences, and in terms of the magnitude of overall RT elevation under adaptation. As indicated, it seems likely that horizontal-vertical differences may be a function of astigmatism. The source of overall differences in RT elevation is more mysterious; although it may be partially a function of individual criterion differences, the near absence of false alarms in all subjects under all conditions suggests that they were operating under a high criterion.

2. Contrast thresholds for this subject were found to be slightly greater for a -45 -deg grating than for a $+45-$ deg grating. No differences were found between his contrast thresholds for horizontal and vertical stimuli, but these were lower than thresholds for the two oblique stimuli.

(Received for publication February 27, 1974; revision received August 19, 1974.) 